\title{
EFEITO DE MACRONUTRIENTES SOBRE O CRESCIMENTO E QUALIDADE DE MUDAS DE CANAFÍSTULA CULTIVADAS EM LATOSSOLO VERMELHO-AMARELO DISTRÓFICO ${ }^{1}$
}

\author{
Cezar Augusto Fonseca e Cruz², Ana Catarina Monteiro Carvalho Mori da Cunha3 \\ Haroldo Nogueira de Paiva ${ }^{4}$ Júlio César Lima Neves ${ }^{5}$
}

\begin{abstract}
RESUMO - Peltophorum dubium (canafístula, angico-cangalha, angico-amarelo) é uma espécie de rápido crescimento, rusticidade e ótima para uso em reflorestamentos mistos de áreas degradadas. Devido à carência de informações sobre a nutrição dessa espécie, o objetivo deste trabalho foi avaliar o efeito de doses de N, P, K, Ca, Mg e S sobre crescimento, produção de biomassa e índices de qualidade de mudas H/D, H/MSPA, MSPA/MSR e IQD, e determinar a dose recomendada destes elementos. No trabalho, realizado em casa de vegetação, foi utilizado Latossolo Vermelho-Amarelo distrófico como substrato. Delimitou-se o experimento por meio de matriz baconiana, avaliando-se três doses dos seis macronutrientes e dois tratamentos adicionais, um com doses de referência e outro sem adição de nutrientes. Adotou-se o delineamento inteiramente casualizado com quatro repetições. Verificou-se, através de análise dos contrastes ortogonais, uma resposta significativa de todas as variáveis estudadas com a aplicação dos macronutrientes. Os nutrientes que mais surtiram efeitos significativos foram o P e o Mg, sendo recomendada dose de $600 \mathrm{mg} / \mathrm{dm}^{3} \mathrm{de} \mathrm{P,} 0,2 \mathrm{cmol}_{\mathrm{c}} / \mathrm{dm}^{3} \mathrm{de} \mathrm{Mg}$, e doses mínimas de $50 \mathrm{mg} / \mathrm{dm}^{3} \mathrm{de} \mathrm{K}$ e $0,8 \mathrm{cmol} / \mathrm{dm}^{3}$ de Ca. Não foi verificada resposta à aplicação de $\mathrm{N}$ e $\mathrm{S}$, para nenhuma das características avaliadas, o que indica que a espécie tem baixo requerimento por estes nutrientes.
\end{abstract}

Palavras-chave: Nutrição de plantas, Características morfológicas e Peltophorum dubium.

\section{EFFECT OF MACRONUTRIENTS ON THE GROWTH AND QUALITY OF Peltophorum dubium SEEDLINGS GROWN IN DYSTROPHYCOUS RED YELLOW LATOSOIL}

\begin{abstract}
Peltophorum dubium is a fast growing tree species, rustic and excellent for using in mixed forestation in degraded areas. Due to lack of information on the nutrition of this species, the objective of this work was to evaluate the effect of doses of $N, P, K, C a, M g$ and $S$ on the growth, biomass production and quality indexs of H/D, H/MSPA, MSPA/MSR and IQD seedlings, and to determine the recommended doses of those elements. In this work, carried out in a greenhouse, a dustrophycous red yellow latosoil was used as substratum. The experiment was designed in a Baconian Matrix, evaluating three doses of the six macronutrients and two additional treatments, one with reference doses and the other one without the addition of nutrients. It was used a complete random experimental design with four replicates. It was noted through the orthogonal contrasts, a significant response of all the variables studied with the application of macronutrients. The nutrients with the best significant effects were $P$ and $\mathrm{Mg}$, and the recommended doses were $600 \mathrm{mg} / \mathrm{dm}^{3}$ of $P, 0.2 \mathrm{cmol} / \mathrm{dm}^{3}$ of $\mathrm{Mg}$, and minimum doses of $50 \mathrm{mg} / \mathrm{dm}^{3}$ of $\mathrm{K}$ and $0.8 \mathrm{cmol} / \mathrm{dm}^{3}$ of Ca. It was not verified response to application of $N$ and $S$, for any of the characteristics evaluated, which indicates that the species have a low need of these nutrients.
\end{abstract}

Keywords: Plant nutrition, Morphologic characteristics and Peltophorum dubium.

\footnotetext{
${ }^{1}$ Recebido em 17.04.2009 e aceito para publicação em 02.05.2011.

${ }^{2}$ Instituto Estadual de Florestas, Núcleo Operacional de Florestas, Pesca e Biodiversidade Poços de Caldas. E-mail: <ceaufoc1@gmail.com>. ${ }^{3}$ Instituto Federal de Educação, Ciência e Tecnol. do Sudeste de Minas Gerais, IFSEMG, Brasil. E-mail: <catarina_mori@yahoo.com.br> . ${ }^{4}$ Departamento de Engenharia Florestal, Universidade Federal de Viçosa, MG, Brasil. E-mail: <hnpaiva@ufv.br>.

${ }^{5}$ Departamento de Solos, Universidade Federal de Viçosa, MG, Brasil. E-mail: <julio_n2003@yahoo.com.br>.
} 


\section{INTRODUÇÃO}

A canafístula (Peltophorum dubium (Spreng.) Taub.) é uma espécie florestal nativa e com ampla dispersão geográfica, desempenhando papel pioneiro nas áreas abertas, em capoeiras e matas degradadas (MACHIORI, 1997; CARVALHO, 2003).

Carvalho (2003) classifica a espécie como secundária inicial, com crescimento rápido e relativamente tolerante ao frio. O uso da canafístula é recomendável para a arborização urbana e cercas divisórias de diversas propriedades (VENTURIN et al., 1999). Devido ao seu rápido crescimento e rusticidade, é ótima para a composição de reflorestamentos mistos de áreas degradadas de preservação permanente (LORENZI, 1992).

A degradação de pastagens é um problema comum em algumas propriedades rurais de Minas Gerais, sendo causada pelo manejo inadequado. As principais consequências do manejo incorreto são a perda do solo por erosão e baixa retenção da água da chuva, problemas que se agravam em regiões de topografia acidentada como a Zona da Mata mineira e a região do Rio Doce (CARVALHO, 1997).

Nesse sentido, as árvores podem ser usadas para evitar a degradação das pastagens cultivadas com gramíneas, tendo em vista que as espécies florestais apresentam elevado potencial para o controle da erosão; aumentam a capacidade de infiltração de água no solo; bem como aumentam a disponibilidade de nitrogênio e de outros nutrientes no solo, melhorando com isso suas características químicas (CARVALHO, 1997).

No entanto, o sucesso na utilização de espécies florestais nativas, principalmente em projetos de recuperação de áreas degradadas, depende de melhor conhecimento dos seus requerimentos nutricionais (RENÓ et al., 1997), visando aperfeiçoar o sistema de produção de mudas e, consequentemente, aumentar o potencial de sobrevivência e crescimento após o plantio no campo.

O conhecimento dos requerimentos nutricionais e da capacidade de adaptação a ambientes distintos das espécies florestais nativas é ainda incipiente. De acordo com Pezzutti et al. (1999), as espécies florestais possuem exigências nutricionais diferenciadas, sendo, portanto, necessário mais conhecimento dos requerimentos nutricionais para adequada recomendação da adubação, o que possibilitaria maior sucesso na produção de mudas.

Revista Árvore, Viçosa-MG, v.35, n.5, p.983-995, 2011
A necessidade de adubação decorre do fato de que nem sempre o solo é capaz de fornecer todos os nutrientes que as plantas precisam para adequado crescimento. Segundo Gonçalves et al. (2000), as características e quantidades de adubos dependem das necessidades nutricionais da espécie utilizada, da fertilidade do solo, da forma de reação dos adubos com o solo, da eficiência dos adubos e de fatores de ordem econômica.

Para aproveitamento do potencial da canafístula na recuperação de pastagens, faz-se necessário estudos que visem ao estabelecimento de critérios para a recomendação de fertilização, como a determinação de doses críticas dos nutrientes. Diante do exposto, o objetivo desse trabalho foi determinar a dose de macronutrientes que possibilitará maior crescimento e melhor qualidade de mudas de canafistula.

\section{MATERIAL E MÉTODOS}

O estudo foi realizado em casa de vegetação no viveiro de pesquisa do Departamento de Engenharia Florestal da Universidade Federal de Viçosa, Viçosa, MG, no período de janeiro a junho de 2006.

Situado nas coordenadas $20^{\circ} 45^{\prime} \mathrm{S}$ e $42^{\circ} 55^{\prime} \mathrm{W}$ na Zona da Mata de Minas Gerais, o Município de Viçosa possui clima classificado como tropical de altitude, com verão chuvoso e inverno frio e seco, do tipo Cwb, pelo sistema de Köeppen, e possui precipitação média anual de $1.221 \mathrm{~mm}$.

Para a realização do estudo, foram usadas amostras de um Latossolo Vermelho-Amarelo distrófico (argila $60 \%$; silte $9 \%$; areia grossa $14 \%$; areia fina $17 \%$ ), retiradas da camada abaixo de $20 \mathrm{~cm}$ de profundidade, secas ao ar e peneiradas em malha de $4 \mathrm{~mm}$ de diâmetro para, então, serem devidamente caracterizadas quimicamente (Tabela 1).

As amostras de solo utilizadas neste estudo foram provenientes de áreas próximas à Viçosa, de ocorrência predominante na região da Zona da Mata mineira, motivo pelo qual foi escolhido para o experimento.

Os tratamentos foram obtidos segundo matriz baconiana (TURRENT, 1979), na qual um dos nutrientes é fornecido em quantidades variávies, enquanto os outros são mantidos em um nível referencial. Com esta matriz, pretendeu-se avaliar seis nutrientes (nitrogênio, fósforo, potássio, cálcio, magnésio e enxofre) em três 
Tabela 1 - Análise química das amostras de solos utilizadas na produção das mudas antes da aplicação dos tratamentos. Table 1 - Chemical analysis of soil sampels used for the production of the seedlings before the treatments.

\begin{tabular}{|c|c|c|c|c|c|c|c|c|c|c|c|c|}
\hline \multirow{2}{*}{$\begin{array}{l}\text { Solo } \\
\text { LVA distrófico }\end{array}$} & \multirow{2}{*}{$\begin{array}{l}\mathrm{pH} \\
\mathrm{H}_{2} \mathrm{O} \\
5,20\end{array}$} & $\mathrm{P}$ & $\mathrm{K}$ & \multirow{2}{*}{$\begin{array}{l}\mathrm{Ca}^{2+} \\
0,30\end{array}$} & \multirow{2}{*}{$\begin{array}{l}\mathrm{Mg}^{2+} \\
0,00\end{array}$} & \multicolumn{2}{|c|}{$\mathrm{cmol}_{\mathrm{c}} / \mathrm{dm}^{3}$} & \multirow{2}{*}{$\begin{array}{c}\text { SB } \\
0,43\end{array}$} & \multirow{2}{*}{$\begin{array}{c}(\mathrm{T}) \\
2,25\end{array}$} & \multirow{2}{*}{$\begin{array}{l}\mathrm{V} \\
19\end{array}$} & \multirow{2}{*}{$\begin{array}{l}\mathrm{m} \\
0\end{array}$} & \multirow{2}{*}{$\begin{array}{r}\begin{array}{r}\mathrm{MO} \\
\mathrm{dag} / \mathrm{kg}\end{array} \\
1,66\end{array}$} \\
\hline & & 0,80 & 49 & & & 0,00 & 1,82 & & & & & \\
\hline $\begin{array}{l}\text { pH em água, } \mathrm{KCl} \\
\mathrm{P} \text { e K - Extrator } \\
\mathrm{CTC}(\mathrm{T})-\mathrm{Capaci} \\
\mathrm{Ca}^{2+}, \mathrm{Mg}^{2+} \text { e } \mathrm{Al}^{3+} \\
\mathrm{H}+\mathrm{Al}-\text { Extrator }\end{array}$ & $\begin{array}{l}\mathrm{CaCl}_{2}^{-}- \\
\text {hlich } 1 \\
\text { de de tr } \\
\text { Extrato }\end{array}$ & $\begin{array}{l}\text { lação } 1 \\
\text { catiôn } \\
\text { Cl } 1 \mathrm{~m}\end{array}$ & 7,0 & & & $\begin{array}{l}\text { Soma } \\
\text { ndice d } \\
\text { - C. Or } \\
\text { Saturaç }\end{array}$ & $\begin{array}{l}\text { e bases } \\
\text { Saturac } \\
\mathrm{x} 1,724 \\
\mathrm{o} \text { por al }\end{array}$ & $\begin{array}{l}\text { cáveis } \\
\text { por ba } \\
\text { Métod } \\
\text { ínio }\end{array}$ & $\begin{array}{l}\text { S } \\
\text { Walkle }\end{array}$ & ack & & \\
\hline
\end{tabular}

diferentes doses, e ainda dois tratamentos adicionais, sendo um com doses de referência e outro sem adição de nutrientes, totalizando 20 tratamentos, que foram dispostos em delineamento inteiramente casualizado, com quatro repetições. Os tratamentos foram delimitados de maneira que, quando a quantidade de um nutriente estivesse variando, as doses dos demais estariam fixas. A variação das doses e nutrientes em cada tratamento pode ser visualizada na Tabela 2.

A unidade experimental foi constituída por um vaso de polietileno rígido com capacidade de $2,1 \mathrm{dm}^{3}$ de solo e uma muda. A espécie selecionada para a realização deste trabalho foi Peltophorum dubium (canafístula), cujas sementes foram originárias do Laboratório de Análise de Sementes (LASO) da Fundação Estadual de Pesquisa Agropecuária (FEPAGRO) do Rio Grande do Sul.

Foram usadas $2,1 \mathrm{~kg}$ de solo como substrato na produção de mudas e, em seguida, acondicionados em sacos plásticos. Posteriormente, foram adicionadas ao solo doses de corretivos e uma mistura de $\mathrm{CaCO}_{3}$ e $\mathrm{MgCO}_{3}$, nas quantidades definidas pelos tratamentos (Tabela 2), e homogeneizados. Depois da aplicação dos corretivos, seguiu-se um período de incubação de 30 dias, mantendo o teor de água próximo à capacidade de campo.

Decorrido esse período, foram adicionados os demais nutrientes de acordo com as quantidades definidas na Tabela 2. A aplicação de nitrogênio e potássio foi igualmente parcelada em 0, 30, 50, 70 e 90 dias após a semeadura. Os sais usados na adubação foram: $\mathrm{NH}_{4} \mathrm{H}_{2} \mathrm{PO}_{4}$ para o $\mathrm{Ne} \mathrm{P}$; $\mathrm{NH}_{4} \mathrm{NO}_{3}$ para o $\mathrm{N}$; $\left(\mathrm{NH}_{4}\right)_{2} \mathrm{SO}_{4}$ para N e S; $\mathrm{K}_{2} \mathrm{SO}_{4}$ para S e $\mathrm{K}$; $\mathrm{KCl}$ para $\mathrm{K}$ e; $\mathrm{CaCO}_{3}$ e $\mathrm{MgCO}_{3}$ para Ca e $\mathrm{Mg}$.

Antes da semeadura, aplicou-se uma solução de micronutrientes composta por: $0,81 \mathrm{mg} / \mathrm{dm}^{3}$ de boro $\left(\mathrm{H}_{3} \mathrm{BO}_{3}\right), 1,33 \mathrm{mg} / \mathrm{dm}^{3}$ de cobre $\left(\mathrm{CuSO}_{4} \cdot 5 \mathrm{H}_{2} \mathrm{O}\right)$, $0,15 \mathrm{mg} / \mathrm{dm}^{3}$ de molibdênio $\left(\left(\mathrm{NH}_{4}\right)_{6} \mathrm{Mo}_{7} \mathrm{O}_{24} \cdot 4 \mathrm{H}_{2} \mathrm{O}\right)$, $3,66 \mathrm{mg} / \mathrm{dm}^{3}$ de manganês $\left(\mathrm{MnCl}_{2} \cdot \mathrm{H}_{2} \mathrm{O}\right)$ e $4,0 \mathrm{mg} / \mathrm{dm}^{3}$ de zinco $\left(\mathrm{ZnSO}_{4} \cdot 7 \mathrm{H}_{2} \mathrm{O}\right)$, como indicado por Alvarez V. (1974).

Tabela 2 - Tratamentos, obtidos pela matriz baconiana, com as doses dos nutrientes aplicadas no substrato utilizado para a produção das mudas.

Table 2 - Treatments obtained through the Baconian Matrix, with the doses of nutrients applied in the substratum used for the production of the seedlings.

\begin{tabular}{|c|c|c|c|}
\hline TRATAMENTOS & UNIDADES & TRATAMENTOS & UNIDADES \\
\hline$\overline{\mathrm{DR}} *$ & $\mathrm{mg} / \mathrm{dm}^{3} / \mathrm{cmol}_{\mathrm{c}} / \mathrm{dm}^{3}$ & $\mathrm{~K}=200 * *$ & $\mathrm{mg} / \mathrm{dm}^{3}$ \\
\hline Solo sem correção & $-e^{c}$ & $\mathrm{Ca}=0,8 * *$ & $\mathrm{cmol}_{\mathrm{c}} / \mathrm{dm}^{3}$ \\
\hline $\mathrm{N}=50 * *$ & $\mathrm{mg} / \mathrm{dm}^{3}$ & $\mathrm{Ca}=1,2 * *$ & $\mathrm{cmol}_{\mathrm{c}}^{\mathrm{c}} / \mathrm{dm}^{3}$ \\
\hline $\mathrm{N}=150 * *$ & $\mathrm{mg} / \mathrm{dm}^{3}$ & $\mathrm{Ca}=1,4 * *$ & $\mathrm{cmol}^{\mathrm{c}} / \mathrm{dm}^{3}$ \\
\hline $\mathrm{N}=200 * *$ & $\mathrm{mg} / \mathrm{dm}^{3}$ & $\mathrm{Mg}=0,2 * *$ & $\mathrm{cmol}_{\mathrm{c}}^{\mathrm{c}} / \mathrm{dm}^{3}$ \\
\hline $\mathrm{P}=150 * *$ & $\mathrm{mg} / \mathrm{dm}^{3}$ & $\mathrm{Mg}=0,6 * *$ & $\mathrm{cmol}^{\mathrm{c}} / \mathrm{dm}^{3}$ \\
\hline $\mathrm{P}=450 * *$ & $\mathrm{mg} / \mathrm{dm}^{3}$ & $\mathrm{Mg}=0,8 * *$ & $\mathrm{cmol}_{\mathrm{c}} / \mathrm{dm}^{3}$ \\
\hline $\mathrm{P}=600 * *$ & $\mathrm{mg} / \mathrm{dm}^{3}$ & $\mathrm{~S}=20 * *$ & $\mathrm{mg} / \mathrm{dm}^{3}$ \\
\hline $\mathrm{K}=50 * *$ & $\mathrm{mg} / \mathrm{dm}^{3}$ & $\mathrm{~S}=60 * *$ & $\mathrm{mg} / \mathrm{dm}^{3}$ \\
\hline $\mathrm{K}=150 * *$ & $\mathrm{mg} / \mathrm{dm}^{3}$ & $\mathrm{~S}=80 * *$ & $\mathrm{mg} / \mathrm{dm}^{3}$ \\
\hline
\end{tabular}

* Valores da dose de referência: $\mathrm{N}=100 \mathrm{mg} / \mathrm{dm}^{3} ; \mathrm{P}=300 \mathrm{mg} / \mathrm{dm}^{3} ; \mathrm{K}=100 \mathrm{mg} / \mathrm{dm}^{3} ; \mathrm{Ca}=1 \mathrm{cmol}_{\mathrm{c}} / \mathrm{dm}^{3} ; \mathrm{Mg}=0,4 \mathrm{cmol}_{\mathrm{c}} / \mathrm{dm}{ }^{3}$; $\mathrm{S}=40 \mathrm{mg} / \mathrm{dm}^{3}$

** Em cada tratamento, apenas o nutriente indicado teve variação na dose, permanecendo a dos demais nutrientes igual à dose de referência.

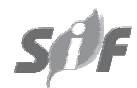

Revista Árvore, Viçosa-MG, v.35, n.5, p.983-995, 2011 
Fez-se a semeadura de 10 sementes diretamente nos vasos. O primeiro raleio foi realizado 15 dias após a emergência das plântulas, deixando-se duas plântulas por vaso, e o segundo aos 30 dias, permanecendo então apenas uma plântula por vaso. O teor de umidade do solo foi controlado diariamente a fim de mantê-lo próximo de $60 \%$ da capacidade de campo.

Aos 120 dias após a semeadura, realizou-se a medição da altura $(\mathrm{H})$, com régua graduada em centímetros, e o diâmetro do colo (DC) das mudas com paquímetro de precisão de 0,01 mm, e deu-se por encerrado o experimento. Em seguida, as plantas foram colhidas e subdivididas em raiz e parte aérea, lavadas em água destilada e postas a secar em estufa com circulação forçada de ar a $60{ }^{\circ} \mathrm{C}$ até que atingissem peso constante.

A determinação do peso de matéria seca de raiz (MSR) e peso de matéria seca da parte aérea (MSPA) foi feita em balança analítica com precisão de 0,01 g, e o peso de matéria seca total (MST) foi obtido pela soma da MSR e da MSPA. Com esses dados, calcularam-se os índices de qualidade de mudas: altura de parte aérea por diâmetro do coleto (H/D), altura de parte aérea por peso de matéria seca de parte aérea (H/MSPA), peso de matéria seca de parte aérea por peso de matéria seca de raiz (MSPA/MSR), bem como o Índice de Qualidade de Dickson (IQD), de acordo com a fórmula (DICKSON et al., 1960):

$$
\mathrm{IQD}=\mathrm{MST} /(\mathrm{H} / \mathrm{D}+\mathrm{MSPA} / \mathrm{MSR})
$$

sendo MST = peso de matéria seca total (g); $\mathrm{H}=$ altura de parte aérea $(\mathrm{cm}) ; \mathrm{D}=$ diâmetro do coleto $(\mathrm{mm})$; MSR = peso de matéria seca de raiz (g); MSPA = peso de matéria seca da parte aérea (g).

Os dados foram interpretados estatisticamente por meio de contrastes ortogonais, análises de variância e regressão, utilizando-se o programa SAEG (Sistema de Análises Estatística e Genética) (EUCLYDES, 1997) e o Statistica. A partir das análises de variância individuais foram estimados o erro experimental e de regressão, e procedeu-se à escolha do modelo com melhor ajuste aos dados, com base no coeficiente de determinação $\left(\mathrm{R}^{2}\right)$. O ajuste das equações de regressão foi feito testando-se os respectivos coeficientes pelo teste “t”, de Student, com base no quadrado médio do resíduo da ANOVA a 1,5 e $10 \%$ de probabilidade. Diante de dois ou mais modelos com coeficientes significativos, optou-se por aquele que apresentou maior $\mathrm{R}^{2} \mathrm{e}$, com essas equações, determinaram-se as doses recomendadas dos macronutrientes a 1, 5 e $10 \%$ de probabilidade.

\section{RESULTADOS}

Verificou-se, pela análise dos contrastes ortogonais que houve resposta significativa de todas as variáveis estudadas à aplicação de doses crescentes dos macronutrientes, em relação ao tratamento sem fertilização, ou seja, a aplicação de fertilizantes contendo N, P, K, Ca, Mg e S possibilitou significativo aumento do crescimento das mudas de canafístula.

Observaram-se baixas médias de crescimento no tratamento sem adição de sais para H, DC, MSPA, MSR e MST (Tabela 3). Os valores médios observados nas diversas características avaliadas, em geral, aumentaram em razão da aplicação de doses crescentes de N, P, K, Ca, Mg e S, à exceção da relação H/MSPA (Tabela 3).

\subsection{Altura da parte aérea (H)}

Em relação ao crescimento em $\mathrm{H}$, somente observouse efeito significativo da aplicação de doses crescentes de macronutrientes para o P (Tabela 4), sendo essa resposta de ordem linear positiva.

Para todos os macronutrientes, as mudas referentes ao tratamento-controle (sem aplicação de fertilizantes) apresentaram crescimento em $\mathrm{H}$ inferior, em média, 3 a 4 vezes, comparados aos maiores valores observados nos diversos tratamentos (Tabela 3).

\subsection{Diâmetro do colo (DC)}

Não foi verificado efeito da adição de macronutrientes no crescimento de DC nas mudas de canafístula, exceto o Mg, cujas doses crescentes proporcionaram reposta linear negativa (Tabela 4).

Neste trabalho, observaram-se valores de DC mínimo de 2,6 e máximo de 7,43 mm (Tabela 3), com maior parte dos valores observados entre 5,0 e $6,0 \mathrm{~mm}$. 
Tabela 3 - Médias para canafístula, em razão da aplicação de doses crescentes de N, P, K e S em mg/dm³ e Ca e Mg em $\mathrm{cmol}_{\mathrm{c}} / \mathrm{dm}^{3}$, das seguintes características: altura (H), diâmetro do coleto (DC), peso de matéria seca da parte aérea (MSPA), peso de matéria seca de raiz (MSR), peso de matéria seca total (MST), altura de parte aérea por diâmetro do coleto (H/D), altura de parte aérea por peso de matéria seca de parte aérea (H/MSPA), peso de matéria seca de parte aérea por peso de matéria seca de raiz (MSPA/MSR) e Índice de Qualidade de Dickson (IQD), aos 120 dias após a semeadura, cultivadas em Latossolo Vermelho-Amarelo distrófico.

Table 3 - Means for Peltophorum dubium, related to the application of increasing doses of $N, P, K$, and $S$ in $\mathrm{mg} / \mathrm{dm}^{3}$ and $\mathrm{Ca}$ and $\mathrm{Mg}$ in $\mathrm{cmol}_{\mathrm{c}} / \mathrm{dm}^{3}$, of the following characteristics: height $(\mathrm{H})$, collar diameter (DC), aerial part dry weight (APDW), root dry weight (RDW), total dry weight (TDW), height of the aerial part per collar diameter (H/D), height of the aerial part per aerial part dry weight (H/APDW), aerial part dry weight per root dry weight(APDW/RDW) and Dickson Quality Index (DQI), at 120 days after sown, grown in dystrophycous red yellow latosoil.

\begin{tabular}{|c|c|c|c|c|c|c|c|c|c|c|}
\hline Nutriente & Dose & $\mathrm{H}(\mathrm{cm})$ & $\mathrm{DC}(\mathrm{mm})$ & MSPA (g) & MSR (g) & MST (g) & $\mathrm{H} / \mathrm{D}$ & H/MSPA & MSPA/MSR & IQD \\
\hline $\mathrm{N}$ & 0 & 7,52 & 2,60 & 0,41 & 0,48 & 0,89 & 2,89 & 18,19 & 0,86 & 0,24 \\
\hline $\mathrm{N}$ & 50 & 17,70 & 4,95 & 2,46 & 1,84 & 4,31 & 3,66 & 7,33 & 1,36 & 0,85 \\
\hline $\mathrm{N}$ & 100 & 21,68 & 5,65 & 3,95 & 2,28 & 6,22 & 3,76 & 5,84 & 1,68 & 1,11 \\
\hline $\mathrm{N}$ & 150 & 24,95 & 5,80 & 4,86 & 2,46 & 7,33 & 4,47 & 5,63 & 1,94 & 1,15 \\
\hline $\mathrm{N}$ & 200 & 21,10 & 5,84 & 3,99 & 2,41 & 6,40 & 3,61 & 5,52 & 1,67 & 1,21 \\
\hline $\mathrm{P}$ & 0 & 7,52 & 2,60 & 0,41 & 0,48 & 0,89 & 2,89 & 18,19 & 0,86 & 0,24 \\
\hline $\mathrm{P}$ & 150 & 16,36 & 5,35 & 2,29 & 1,53 & 3,81 & 3,07 & 8,05 & 1,49 & 0,85 \\
\hline $\mathrm{P}$ & 300 & 21,68 & 5,65 & 3,95 & 2,28 & 6,22 & 3,76 & 5,84 & 1,68 & 1,11 \\
\hline $\mathrm{P}$ & 450 & 22,15 & 6,14 & 4,30 & 2,37 & 6,67 & 3,63 & 5,27 & 1,81 & 1,24 \\
\hline $\mathrm{P}$ & 600 & 28,80 & 6,80 & 6,77 & 3,14 & 9,91 & 4,28 & 4,84 & 2,24 & 1,56 \\
\hline K & 0 & 7,52 & 2,60 & 0,41 & 0,48 & 0,89 & 2,89 & 18,19 & 0,86 & 0,24 \\
\hline K & 50 & 24,18 & 7,05 & 5,84 & 3,16 & 8,99 & 3,43 & 4,15 & 1,87 & 1,72 \\
\hline K & 100 & 21,68 & 5,65 & 3,95 & 2,28 & 6,22 & 3,76 & 5,84 & 1,68 & 1,11 \\
\hline K & 150 & 28,73 & 7,32 & 6,44 & 3,35 & 9,80 & 3,98 & 4,49 & 1,91 & 1,67 \\
\hline $\mathrm{K}$ & 200 & 24,62 & 5,98 & 4,59 & 2,53 & 7,11 & 4,10 & 5,56 & 1,80 & 1,20 \\
\hline $\mathrm{Ca}$ & 0 & 7,52 & 2,60 & 0,41 & 0,48 & 0,89 & 2,89 & 18,19 & 0,86 & 0,24 \\
\hline $\mathrm{Ca}$ & 0,8 & 23,88 & 6,65 & 5,54 & 3,08 & 8,62 & 3,60 & 4,36 & 1,81 & 1,61 \\
\hline $\mathrm{Ca}$ & 1,0 & 21,68 & 5,65 & 3,95 & 2,28 & 6,22 & 3,76 & 5,84 & 1,68 & 1,11 \\
\hline $\mathrm{Ca}$ & 1,2 & 26,53 & 5,79 & 4,58 & 2,23 & 6,81 & 4,61 & 5,79 & 2,10 & 1,03 \\
\hline $\mathrm{Ca}$ & 1,4 & 24,92 & 6,14 & 6,00 & 3,12 & 9,11 & 4,11 & 4,41 & 1,92 & 1,53 \\
\hline $\mathrm{Mg}$ & 0 & 7,52 & 2,60 & 0,41 & 0,48 & 0,89 & 2,89 & 18,19 & 0,86 & 0,24 \\
\hline $\mathrm{Mg}$ & 0,2 & 26,80 & 7,43 & 7,77 & 4,23 & 11,99 & 3,62 & 3,47 & 1,84 & 2,22 \\
\hline $\mathrm{Mg}$ & 0,4 & 21,68 & 5,65 & 3,95 & 2,28 & 6,22 & 3,76 & 5,84 & 1,68 & 1,11 \\
\hline $\mathrm{Mg}$ & 0,6 & 28,43 & 7,24 & 7,03 & 3,42 & 10,45 & 3,97 & 4,13 & 2,06 & 1,75 \\
\hline $\mathrm{Mg}$ & 0,8 & 20,50 & 5,55 & 3,43 & 2,22 & 5,65 & 3,81 & 6,61 & 1,65 & 1,18 \\
\hline S & 0 & 7,52 & 2,60 & 0,41 & 0,48 & 0,89 & 2,89 & 18,19 & 0,86 & 0,24 \\
\hline S & 20 & 29,65 & 7,18 & 6,90 & 3,61 & 10,51 & 4,11 & 4,29 & 1,96 & 1,78 \\
\hline S & 40 & 21,68 & 5,65 & 3,95 & 2,28 & 6,22 & 3,76 & 5,84 & 1,68 & 1,11 \\
\hline S & 60 & 28,00 & 6,81 & 6,61 & 3,32 & 9,92 & 4,09 & 4,39 & 1,96 & 1,63 \\
\hline S & 80 & 24,58 & 6,72 & 5,60 & 2,97 & 8,57 & 3,65 & 4,76 & 1,90 & 1,55 \\
\hline
\end{tabular}

\subsection{Produção de matéria seca}

A aplicação de doses crescentes de N, K, Ca e S não acarretou efeito sobre a produção de matéria seca das plantas, tanto de massa seca da parte aérea quanto de massa seca da raiz e total. No entanto, a aplicação de doses de $\mathrm{P}$ resultou em resposta linear crescente para MSPA, MSR e MST (Tabela 4). Já para o Mg, observou-se resposta oposta, ou seja, efeito linear negativo para as mesmas características em resposta à adição de doses crescentes do nutriente ao substrato (Tabela 4). 
Tabela 4 - Estimativas geradas em razão da aplicação de doses crescentes de N, P, K e S em mg/dm e Ca e Mg em cmol / dm3, das seguintes características em mudas de canafistula: altura $(\mathrm{H})$, diâmetro do coleto (DC), peso de matéria seca da parte aérea (MSPA), peso de matéria seca de raiz (MSR), peso de matéria seca total (MST), aos 120 dias após a semeadura, cultivadas em Latossolo Vermelho-Amarelo distrófico.

Table 4-Generated estimations related to the application of increasing doses of $N, P, K$, and $S$ in $\mathrm{mg} / \mathrm{dm}^{3}$ and Ca and $\mathrm{Mg}$ in $\mathrm{cmol}_{\mathrm{c}} / \mathrm{dm}^{3}$, of the following characteristics in Peltophorum dubium seedlings: height $(\mathrm{H})$, collar diameter (CD), aerial part dry weight (APDW), root dry weight (RDW), total dry weight (TDW), at 120 days after sown, grown in dystrophycous red yellow latosoil.

\begin{tabular}{|c|c|c|c|c|}
\hline Característica & Nutriente & Equação & $\mathrm{R}^{2}$ & Dose recomendada \\
\hline \multirow[t]{6}{*}{$\mathrm{H}$} & $\mathrm{N}$ & $\hat{Y}=\bar{Y}=21,36$ & & 50 \\
\hline & $P$ & $\hat{Y}=12,8125+0,0252 * \mathrm{X}$ & 91,63 & 600 \\
\hline & $\mathrm{K}$ & $\hat{Y}=\bar{Y}=24,80$ & & 50 \\
\hline & $\mathrm{Ca}$ & $\hat{Y}=\bar{Y}=24,25$ & & 0,8 \\
\hline & Mg & $\hat{Y}=\bar{Y}=24,35$ & & 0,2 \\
\hline & $\mathrm{S}$ & $\hat{Y}=\bar{Y}=25,98$ & & 20 \\
\hline \multirow[t]{6}{*}{$\overline{\mathrm{DC}}$} & $\mathrm{N}$ & $\hat{Y}=\bar{Y}=5,56$ & & 50 \\
\hline & $\mathrm{P}$ & $\hat{Y}=\bar{Y}=5,99$ & & 150 \\
\hline & $\mathrm{K}$ & $\hat{Y}=\bar{Y}=6,50$ & & 50 \\
\hline & Ca & $\hat{Y}=\bar{Y}=6,06$ & & 0,8 \\
\hline & $\mathrm{Mg}$ & $\hat{Y}=7,475-2,02 * X$ & 26,91 & 0,2 \\
\hline & $\mathrm{s}$ & $\hat{Y}=\bar{Y}=6,59$ & & 20 \\
\hline \multirow[t]{6}{*}{ MSPA } & $\mathrm{N}$ & $\hat{Y}=\bar{Y}=3,82$ & & 50 \\
\hline & $\mathrm{P}$ & $\hat{Y}=08763+0,0092 * X$ & 92,66 & 600 \\
\hline & $\mathrm{K}$ & $\hat{Y}=\bar{Y}=5,21$ & & 50 \\
\hline & $\mathrm{Ca}$ & $\hat{Y}=\bar{Y}=5,02$ & & 0,8 \\
\hline & $\mathrm{Mg}$ & $\hat{Y}=8,0263-4,97 * X$ & 34,86 & 0,2 \\
\hline & $\mathrm{S}$ & $\hat{Y}=\bar{Y}=5,77$ & & 20 \\
\hline \multirow[t]{6}{*}{ MSR } & $\mathrm{N}$ & $\hat{Y}=\bar{Y}=2,25$ & & 50 \\
\hline & $\mathrm{P}$ & $\hat{Y}=1,0913+0,0033^{*} X$ & 93,15 & 600 \\
\hline & $\mathrm{K}$ & $\hat{Y}=\bar{Y}=2,83$ & & 50 \\
\hline & $\mathrm{Ca}$ & $\hat{Y}=\bar{Y}=2,68$ & & 0,8 \\
\hline & Mg & $\hat{Y}=4,2525-2,4363 * * X$ & 42,33 & 0,2 \\
\hline & $\mathrm{s}$ & $\hat{Y}=\bar{Y}=3,05$ & & 20 \\
\hline \multirow[t]{6}{*}{ MST } & $\mathrm{N}$ & $\hat{Y}=\bar{Y}=6,07$ & & 50 \\
\hline & $\mathrm{P}$ & $\hat{Y}=1,9675+0,0125^{*} X$ & 93,04 & 600 \\
\hline & $\mathrm{K}$ & $\hat{Y}=\bar{Y}=8,03$ & & 50 \\
\hline & $\mathrm{Ca}$ & $\hat{Y}=\bar{Y}=7,69$ & & 0,8 \\
\hline & $\mathrm{Mg}$ & $\hat{Y}=12,2787-7,4063^{* *} X$ & 37,47 & 0,2 \\
\hline & $\mathrm{S}$ & $\hat{Y}=\bar{Y}=8,81$ & & 20 \\
\hline
\end{tabular}

***Significativo a $1 \%$ de probabilidade, **significativo a $5 \%$ de probabilidade e *significativo a $10 \%$ de probabilidade.

\section{4. Índices de qualidade de mudas}

\subsubsection{Relação entre a altura da parte aérea e 0 diâmetro do coleto (H/D)}

Quanto à relação H/D, somente foram observadas respostas significativas para $\mathrm{P}$ e Ca (Tabela 5). Para $\mathrm{P}$, verificou-se efeito linear positivo. Já para Ca, observou-se resposta explicada por um modelo de ordem quadrática.

\subsubsection{Relação entre altura e peso de matéria seca da parte aérea (H/MSPA)}

Para a relação H/MSPA, observou-se que os tratamentos com doses crescentes de K, Ca e Mg proporcionaram resposta explicada por modelo linear positivo para Ke Mg e modelo hiperbólico quadrático para Ca (Tabela 5). Para os demais macronutrientes, não foi observado efeito da aplicação no substrato sobre a relação H/MSPA.

Revista Árvore, Viçosa-MG, v.35, n.5, p.983-995, 2011 
Tabela 5 - Estimativas geradas em razão da aplicação de doses crescentes de N, P, K e S em mg dm ${ }^{-3}$ e Ca e Mg em cmol dm-3 das seguintes características em mudas de canafistula: altura de parte aérea por diâmetro do coleto (H/D), altura de parte aérea por peso de matéria seca de parte aérea (H/MSPA), peso de matéria seca de parte aérea por peso de matéria seca de raiz (MSPA/MSR) e Índice de Qualidade de Dickson (IQD), aos 120 dias após a semeadura, cultivadas em Latossolo Vermelho-Amarelo distrófico.

Table 5 - Generated estimations related to the application of increasing doses of $N, P, K$, and $S$ in $\mathrm{mg} / \mathrm{dm}^{3}$ and Ca and $\mathrm{Mg}$ in $\mathrm{cmol}_{\mathrm{c}} / \mathrm{dm}^{3}$, of the following characteristics in Peltophorum dubium seedlings: height of the aerial part per collar diameter $(H / D)$, height of the aerial part per aerial part dry weight (H/APDW), aerial part dry weight per root dry weight(APDW/RDW) and Dickson Quality Index (DQI), at 120 days after sown, grown in dystrophycous red yellow latosoil.

\begin{tabular}{|c|c|c|c|c|}
\hline Característica & Nutriente & Equação & $\mathrm{R}^{2}$ & Dose recomendada \\
\hline \multirow[t]{6}{*}{$\mathrm{H} / \mathrm{D}$} & $\mathrm{N}$ & $\hat{Y}=\bar{Y}=3,88$ & & 50 \\
\hline & $\mathrm{P}$ & $\hat{Y}=2,8136+0,0023 * X$ & 82,16 & 150 \\
\hline & K & $\hat{Y}=\bar{Y}=3,82$ & & 50 \\
\hline & $\mathrm{Ca}$ & $\hat{Y}=-2,1274+10,364 * X-4,1719 * X^{2}$ & 65,87 & 0,8 \\
\hline & Mg & $\hat{Y}=\bar{Y}=3,79$ & & 0,2 \\
\hline & $\mathrm{S}$ & $\hat{Y}=\bar{Y}=3,90$ & & 20 \\
\hline \multirow[t]{6}{*}{ H/MSPA } & $\mathrm{N}$ & $\hat{Y}=\bar{Y}=6,08$ & & 50 \\
\hline & $\mathrm{P}$ & $\hat{Y}=\bar{Y}=6,00$ & & 150 \\
\hline & $\mathrm{K}$ & $\hat{Y}=4,2863+0,0058 * * X$ & 20,86 & 50 \\
\hline & $\mathrm{Ca}$ & $\hat{Y}=-15,707+39,408 * * X-17,891 * * X^{2}$ & 99,92 & 0,8 \\
\hline & Mg & $\hat{Y}=3,0845+3,8575 * * X$ & 46,61 & 0,2 \\
\hline & $\mathrm{S}$ & $\hat{Y}=\bar{Y}=4,82$ & & 20 \\
\hline \multirow[t]{6}{*}{ MSPA/MSR } & $\mathrm{N}$ & $\hat{Y}=\bar{Y}=1,66$ & & 50 \\
\hline & $\mathrm{P}$ & $\hat{Y}=\bar{Y}=1,208+0,0016 * X$ & 94,01 & 495 \\
\hline & $\mathrm{K}$ & $\hat{Y}=\bar{Y}=1,82$ & & 50 \\
\hline & $\mathrm{Ca}$ & $\hat{Y}=\bar{Y}=1,88$ & & 0,8 \\
\hline & Mg & $\hat{Y}=\bar{Y}=1,81$ & & 0,2 \\
\hline & $\mathrm{S}$ & $\hat{Y}=\bar{Y}=1,88$ & & 20 \\
\hline \multirow[t]{6}{*}{ IQD } & $\mathrm{N}$ & $\hat{Y}=\bar{Y}=1,08$ & & 50 \\
\hline & $\mathrm{P}$ & $\hat{Y}=\bar{Y}=1,19$ & & 150 \\
\hline & $\mathrm{K}$ & $\hat{Y}=1,6713-0,002 * X$ & 17,96 & 50 \\
\hline & $\mathrm{Ca}$ & $\hat{Y}=\bar{Y}=1,32$ & & 0,8 \\
\hline & Mg & $\hat{Y}=2,1904-1,2498 * X$ & 37,91 & 0,2 \\
\hline & $\mathrm{S}$ & $\hat{Y}=\bar{Y}=1,52$ & & 20 \\
\hline
\end{tabular}

***Significativo a $1 \%$ de probabilidade, $* *$ significativo a $5 \%$ de probabilidade e $*$ significativo a $10 \%$ de probabilidade.

\subsubsection{Relação entre peso de matéria seca da parte aérea e peso de matéria seca de raiz (MSPA/MSR)}

Houve respostas significativas da relação MSPA/ MSR somente para os tratamentos com doses crescentes de $\mathrm{P}$, sendo obtido efeito linear positivo e determinada dose recomendada em $495 \mathrm{mg} / \mathrm{dm}^{3}$ (Tabela 5). De modo geral, os valores da relação MSPA/MSR variaram entre 0,86 e 2,24 com grande concentração entre 1,7 e 1,9 (Tabela 3).

\subsection{4. Índice de qualidade de mudas de Dickson (IQD)}

A aplicação de tratamentos com doses de macronutrientes resultou em resposta significativa para os nutrientes K e Mg (Tabela 5), e em ambos foi observado efeito linear negativo sobre o índice IQD. Em relação aos demais nutrientes avaliados neste experimento, não foram observados efeitos da sua aplicação sobre o referido índice nas mudas de canafístula.

Revista Árvore, Viçosa-MG, v.35, n.5, p.983-995, 2011 


\section{DISCUSSÃO}

De modo geral, as baixas médias de crescimento observadas para H, DC, MSPA, MSR e MST no tratamento sem adição de sais indicam que os teores de nutrientes originalmente do solo utilizado como substrato para a produção de mudas de canafístula são baixos. Essas pequenas médias de crescimento eram esperadas, tendo em vista que os nutrientes minerais desempenham diversas funções na planta e, consequentemente, a omissão de qualquer um deles acarreta diminuição no ritmo de crescimento, bem como pode ser prejudicial à qualidade das mudas produzidas.

\subsection{Altura da parte aérea}

Respostas significativas à aplicação de N também foram observadas por Marques et al. (2006a,b, 2009), que observaram efeito positivo do $\mathrm{N}$ sobre o crescimento em H de mudas de Mimosa caesalpinaefolia, Dalbergia nigra e Piptadenia gonoacantha, respectivamente. As repostas observadas por esses autores são contrárias às observadas neste estudo, indicando que a necessidade do $\mathrm{N}$ pode ter sido suprida pelo teores originais do nutriente no solo, ou que a espécie possui baixa exigência nutricional de N. Respostas semelhantes foram obtidas por Gonçalves et al. (2008) com mudas de Anadenathera macrocarpa, em condições experimentais iguais às deste experimento.

Para a obtenção de $\mathrm{H}$ máxima em mudas de canafístula, a dose crítica de P está acima de 600 $\mathrm{mg} / \mathrm{dm}^{3}$. Resultados semelhantes aos observados neste estudo foram obtidos por Reis et al. (1997) e Gonçalves et al. (2008), que também verificaram resposta significativa à aplicação de $\mathrm{P}$, obtendo resposta quadrática para Dalbergia nigra e linear positiva para Anadenanthera macrocarpa sobre o crescimento em $\mathrm{H}$, respectivamente.

Balieiro et al. (2001) também verificaram, em mudas de Acacia auriculiformis, ausência de efeito da aplicação de $\mathrm{K}$ ao substrato, e melhor desempenho de crescimento em $\mathrm{H}$ das mudas foi observado nos tratamentos com ausência do nutriente.

Quanto à aplicação de Ca ao substrato, Cruz et al. (2004) verificaram ausência de efeito sobre o crescimento em H de mudas de Tabebuia impetiginosa à adição do nutriente, resultado semelhante ao verificado neste estudo. Já para o magnésio, Venturin et al. (1999) observaram também, para canafístula, que o nutriente mostrou-se limitante ao crescimento em $\mathrm{H}$ das mudas, sendo significativa a resposta à aplicação do nutriente. Os resultados encontrados por Venturin et al. (1999) são indicativo de que os teores de $\mathrm{Mg}$ do solo usado como substrato foram suficientes para a produção de mudas de canafístula, tendo em vista que foi observada, neste estudo, ausência de efeito da aplicação do nutriente.

Neste estudo, não foi verificado efeito da aplicação de S sobre o crescimento em $\mathrm{H}$ das mudas de canafístula. No entanto, Balieiro et al. (2001) observaram para Acacia holosericea resposta positiva, enquanto Reis et al. (1997), com Dalbergia nigra, observaram resposta negativa.

De acordo com Paiva e Gomes (2000), mudas de espécies arbóreas estão aptas ao plantio no campo quando a $\mathrm{H}$ da parte aérea estiver entre 15 e $30 \mathrm{~cm}$. Ao final desse experimento, na maioria dos tratamentos nos quais foram aplicadas as doses crescentes de nutrientes, verificou-se que os valores médios de $\mathrm{H}$ encontram-se entre os supramencionados, podendo-se afimar assim que as mudas de canafístula, segundo esse critério, estariam aptas ao plantio no campo.

\subsection{Diâmetro do coleto}

Segundo Souza et al. (2006), a avaliação do DC é de fundamental importância na avaliação do potencial da muda para sobrevivência e crescimento após o plantio. De acordo com Carneiro (1983), plantas com maior DC apresentam maior sobrevivência, especialmente pela maior capacidade de formação e de crescimento das novas raízes.

Gonçalves et al. (2008), de modo semelhante ao observado neste experimento, verificaram que mudas de Anadenanthera macrocarpa não tiveram o crescimento em DC afetado em razão da adição de N, enquanto Cruz et al. (2006) e Marques et al. (2006a,b, 2009) observaram efeito significativo para o DC quando da adição de adubação nitrogenada para Samanea inopinata, Mimosa caesalpinaefolia, Dalbergia nigra e Piptadenia gonoacantha, respectivamente.

Respostas contrárias às obtidas neste estudo foram encontradas por Venturin et al. (1999) e Gonçalves et al. (2008), que destacaram a importância de P no meio de cultivo para o crescimento de mudas de Peltophorum dubium e Anadenanthera macrocarpa, respectivamente, 
observando que a espécie é muito exigente em fósforo e a sua ausência é prejudicial ao adequado crescimento em DC das mudas.

Balieiro et al. (2001) também verificaram, para Acacia auriculiformis, que o crescimento em DC não foi afetado pela aplicação de K. Já para Dalbergia nigra e Anadenanthera macrocarpa, Reis et al. (1997) e Gonçalves et al. (2008), respectivamente, verificaram efeito positivo da aplicação de K sobre o crescimento em DC das mudas

Gonçalves et al. (2008) obtiveram respostas semelhantes às observadas nas mudas de canafístula em relação ao crescimento em DC, ou seja, os autores não observaram efeito significativo da aplicação de Ca sobre as mudas de Anadenanthera macrocarpa. Já Venturin et al. (1999) observaram, para canafístula, menor crescimento em DC devido à omissão de Ca no meio de crescimento.

Para o Mg, observou-se efeito linear negativo sobre o crescimento do DC das mudas, ou seja, à medida que as doses de $\mathrm{Mg}$ aumentaram, houve queda nos valores de DC. Isso indica que, em doses maiores deste nutriente, a característica DC foi prejudicada. Cabe ressaltar, entretanto, que a não adição deste nutriente é prejudicial ao crescimento das mudas de canafístula, ou seja, a dose crítica deste nutriente está abaixo da menor dose testada $\left(0,2 \mathrm{cmol} / \mathrm{dm}^{3}\right)$, mas acima do teor original de Mg no solo usado como substrato. Em contrapartida, Gonçalves et al. (2008) não verificaram efeito do Mg sobre o crescimento em DC das mudas de Anadenanthera macrocarpa nas mesmas condições experimentais, denotando as diferentes exigências nutricionais entre as espécies florestais.

À semelhança do verificado neste trabalho, mudas de Myracrodruon urundeuva (MENDONÇA et al., 1999) não apresentaram, sobre o crescimento em DC, resposta significativa quando submetidas à fertilização com S.

As divergências entre os resultados encontrados em literatura e os apresentados neste trabalho podem estar relacionadas com a metodologia de produção de mudas ou mesmo com as diferentes exigências nutricionais das espécies, o que pode justificar a diferença observada entre os diferentes experimentos.

\subsection{Produção de matéria seca}

De acordo com Carneiro (1995), ao se referir ao peso de matéria seca como parâmetro de qualidade das mudas, deve-se considerar uma compartimentalização em raízes, parte aérea e total, e a sobrevivência e o crescimento inicial das mudas estão intimamente relacionados com o peso de matéria seca delas. Neste estudo, as mudas de canafístula apresentaram respostas diferenciadas e significativas à aplicação dos macronutrientes em cada um dos três compartimentos delimitados.

Contrariando as respostas observadas para mudas de canafístula, Cruz et al. (2006) observaram efeito significativo da adubação nitrogenada sobre a MSPA de Samanea inopinata. Entretanto, respostas análogas foram observadas por Nicoloso et al. (2001) em mudas de Apuleia leiocarpa. No que tange à MSR, Mendonça et al. (1999) também constataram, para Myracrodruon urundeuva, ausência de efeito do N. No entanto, Cruz et al. (2006) observaram resposta significativa da aplicação de N para Samanea inopinata sobre a produção de MSR.

A aplicação de $\mathrm{P}$ resultou em resposta linear positiva para a produção de matéria seca em todos os compartimentos, indicando que a obtenção dos pontos máximos dessas caraterísticas devem ser alcançados com a aplicação de doses superiores a $600 \mathrm{mg} / \mathrm{dm}^{3}$.

Resultados similares aos observados nesse trabalho foram obtidos por Fernandes et al. (2002), Ceconi et al. (2006), Ceconi et al. (2007), Fernandes et al. (2007) e Gomes et al. (2008), com mudas de Cordia goeldiana, Luehea divaricata, Ilex paraguariensis, Cordia goeldiana e Apuleia leiocarpa, respectivamente, ou seja, a aplicação de $\mathrm{P}$ resultou em incremento significativo na produção de matéria seca das plantas.

Fernandes et al. (2000) determinaram para Ceiba speciosa dose crítica de $267,0 \mathrm{mg} / \mathrm{dm}^{3}$ de $\mathrm{P}$ para a obtenção de máximo em crescimento MSPA. Esses resultados são bem inferiores à tendência observada para canafístula, ou seja, esta apresenta-se mais exigente nutricionalmente em $\mathrm{P}$ do que a espécie anterior no que se refere à MSPA. Ainda, Fernandes et al. (2002), de maneira semelhante ao observado nesse estudo, verificaram respostas lineares crescentes à aplicação de doses de $\mathrm{P}$ ao substrato para MSR e MST em Cordia goeldiana.

A adição de doses crescentes de K não aumentou a MSPA das mudas de canafístula. Entretanto, Venturin et al. (1999), estudando a mesma espécie, afirmaram que a adubação potássica é importante para uma adequada produção de MSPA. Cabe ressaltar que o solo usado como subtrado para a produção das

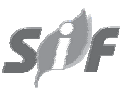

Revista Árvore, Viçosa-MG, v.35, n.5, p.983-995, 2011 
mudas foi diferente entre os experimentos, o que justifica a divergência dos resultados, pois o solo usado pelos referidos autores apresentava teor original de K quatro vezes menor que o solo usado neste estudo. Em relação à MSR, Venturin et al. (1999), semelhante ao observado neste estudo, ressaltaram que a omissão de $\mathrm{K}$ do meio de crescimento não afetou a MSR das plantas. Em contrapartida, para mudas de Myracrodruon urundeuva, Mendonça et al. (1999) observaram que a omissão de $\mathrm{K}$ proporcionou menor MSR.

Reis et al. (1997) também não verificaram efeito da calagem sobre a produção de matéria seca em mudas de Dalbergia nigra. Já para o Mg, foram observados efeitos significativos sobre a produção de matéria seca; o mesmo foi observado por Cruz et al. (2004) em mudas de Tabebuia impetiginosa sob influência da elevação de saturação por bases.

Venturin et al. (1999), trabalhando com a mesma espécie utilizada neste estudo, verificaram que ela apresenta elevada exigência nutricional em Mg, sendo essa resposta contrária à observada neste estudo. Contudo, a dose testada pelos autores foi similar à menor dose testada neste experimento. Isto é mais um indicativo de que a dose crítica, visando maior crescimento das mudas de canafístula, está abaixo da menor dose aplicada $\left(0,2 \mathrm{cmol}_{\mathrm{c}} / \mathrm{dm}^{3} \mathrm{de} \mathrm{Mg}\right)$.

A ausência de resposta à aplicação de S sobre a produção de matéria seca também foi verificada por Mendonça et al. (1999) em mudas de Myracrodruon urundeuva, tanto sobre a MSPA quanto sobre a MSR. Entretanto, respostas significativas à aplicação de $\mathrm{S}$ sobre a produção de matéria seca também foram observadas por Venturin et al. (1999) e Balieiro et al. (2001) em mudas de Peltophorum dubium e Acacia holosericea, respectivamente.

\section{4. Índices de qualidade de mudas}

\subsubsection{Relação entre a altura da parte aérea e o diâmetro do coleto (H/D)}

De acordo com Gomes (2001), em razão da facilidade de medição tanto da H quanto do DC e por ser um método não destrutivo, a relação entre esses parâmetros pode ser considerada e aplicada a muitas das espécies florestais, constituindo-se num dos mais importantes parâmetros morfológicos para estimar o crescimento das mudas após o plantio definitivo no campo. Ainda de acordo com esse autor, o valor resultante da altura da parte aérea da muda pelo seu respectivo diâmetro do coleto exprime um equilíbrio de crescimento.

Verificou-se resposta não siginificativa para a relação $\mathrm{H} / \mathrm{D}$ para todos os macronutrientes, exceto para o $\mathrm{P}$ e $\mathrm{Ca}$, sendo similares às observadas por Cruz et al. (2004), Bernardino et al. (2007) e Souza et al. (2008) para Tabebuia impetiginosa, Dalbergia nigra e Machaerium nictitans, respectivamente, submetida à elevação da saturação por bases, bem como para Samanea inopinata submetidas à adubação nitrogenada (CRUZ et al., 2006). No entanto, Chaves et al. (2006) observaram reposta significativa para a relação H/D em mudas de Anadenanthera macrocarpa produzidas em substrato fertilizado com $\mathrm{N}$.

De acordo com Carneiro (1983), este índice de qualidade de mudas é muito importante e quanto menor for seu valor, maior será a capacidade de sobrevivência e estabelecimento das mudas após seu plantio definitivo no campo. Nesse sentido, observa-se que os nutrientes $\mathrm{P}$ e Ca exerceram efeito negativo sobre a referida característica, sendo recomendada a menor dose testada de cada nutriente. Ainda, cabe ressaltar que há tendência de o menor valor a ser atingido por este índice ocorrer para uma dose abaixo da menor de todos os macronutrientes.

\subsubsection{Relação entre altura e peso de matéria seca da parte aérea (H/MSPA)}

A relação H/MSPA não é comumente usado para avaliar o padrão de qualidade de mudas, mas pode ser de grande valia, principalmente para predizer o potencial de sobrevivência da muda no campo (GOMES, 2001). De acordo com esse autor, quanto menor for esse índice, mais lignificada está a muda e maior a sua capacidade de sobrevivência no campo.

O melhor valor da relação H/MSPA variou entre os tratamentos aplicados. Para os tratamentos com $\mathrm{K}$ e Mg, o menor valor de H/MSPA encontra-se abaixo da menor dose aplicada do nutriente. Já no tratamento com Ca, verificou-se ocorrência de um ponto de máximo, podendo-se interpretar, dessa forma, que o melhor valor de H/MSPA ocorre quando da aplicação de doses maiores ou menores a $1,1 \mathrm{cmol}_{\mathrm{c} /} \mathrm{dm}^{3}$.

Trabalhando com Tabebuia impetiginosa, Cruz et al. (2004) observaram resposta significativa da elevação da saturação por bases sobre a relação 
H/MSPA, enquanto Bernardino et al. (2007) não observaram efeito da elevação de saturação por bases sobre esta relação em mudas de Dalbergia nigra. Respostas significativas para este índice de qualidade de mudas foram encontrados em mudas de Samanea inopinata, Mimosa caesalpiniaefolia e Dalbergia nigra submetidas à adubação nitrogenada por Cruz et al. (2006) e Marques et al. (2006a,b), respectivamente.

\subsubsection{Relação entre peso de matéria seca da parte aérea e peso de matéria seca de raiz (MSPA/MSR)}

A relação entre o peso de matéria seca da parte aérea e do respectivo sistema radicular das mudas é considerada como um índice eficiente e seguro para expressar o padrão de qualidade destas (GOMES, 2001).

Brissette (1984) menciona que, em um encontro de pesquisadores, ficou estabelecido que um valor igual a 2,0 seria a melhor relação de MSPA/MSR de uma mesma planta. De forma geral, pode-se observar que os valores médios para as maiores doses de cada nutriente (Tabela 3) se encontram próximos do valor tido como ideal $(2,0)$, permitindo inferir que, por esse índice e para essas doses, as mudas de canafístula alcançaram um bom padrão de qualidade.

\subsection{4. Índice de qualidade de mudas de Dickson (IQD)}

O IQD é uma fórmula balanceada onde estão incluídas as relações dos parâmetros morfológicos (GOMES, 2001), desenvolvido enquanto Dickson et al. (1960)estudavam o comportamento de mudas de Picea glauca e Pinus monficola. Segundo Gomes (2001), quanto maior o valor do índice, melhor o padrão de qualidade das mudas.

Verificou-se que houve respostas não significativas para todos os macronutrientes em relação ao índice IQD, exceto para $\mathrm{K}$ e $\mathrm{Mg}$, sendo essas respostas semelhantes às encontradas por Bernardino et al. (2007) em mudas de Dalbergia nigra, submetidas à elevação de saturação por bases. Em contrapartida, Marques et al. (2006a, b, 2009) observaram efeito significativo da aplicação de N sobre o referido índice em mudas de Mimosa caesalpiniaefolia, Dalbergia nigra e Piptadenia gonoacantha, respectivamente. Neste estudo, verificou-se que quanto maiores as doses aplicadas, menor foi o valor desse índice. Isto indica que a espécie é pouco exigente em $\mathrm{K}$ e Mg.

\section{CONCLUSÃO}

As mudas de canafístula respondem, de modo significativo, à adição de macronutrientes, sendo a fertilização importante para o adequado crescimento e qualidade de mudas da espécie.

Com base nos dados de matéria seca total, que tem sido considerada como a característica que melhor reflete a produção, as doses de macronutrientes recomendadas para a produção de mudas de canafístula são: $50 \mathrm{mg} / \mathrm{dm}^{3}$ de $\mathrm{N} ; 600 \mathrm{mg} / \mathrm{dm}^{3}$ de P; $50 \mathrm{mg} / \mathrm{dm}^{3} \mathrm{de}$ $\mathrm{K} ; 0,8 \mathrm{cmol}_{\mathrm{c}} / \mathrm{dm}^{3} \mathrm{de} \mathrm{Ca} ; 0,2 \mathrm{cmol} / \mathrm{dm}^{3} \mathrm{de} \mathrm{Mg}$ e $20 \mathrm{mg} / \mathrm{dm}^{3}$ de S, utilizando Latossolo Vermelho-Amarelo distrófico como substrato.

As mudas de canafístula mostraram-se bastante exigentes em $\mathrm{P}$ e menos exigentes em Mg. Recomendam-se estudos adicionais com os nutrientes $\mathrm{N}$ e $\mathrm{S}$, com doses inferiores às menores doses testadas, tendo em vista que, segundo dados deste estudo, a melhor dose desses nutrientes encontra-se abaixo da menor dose testada.

\section{AGRADECIMENTOS}

Os autores agradecem ao CNPq, pela concessão de bolsa de estudos e pela bolsa de produtividade em pesquisa, e ao projeto PRODETAB 130-02/01, pelo financiamento do presente trabalho.

\section{REFERÊNCIAS}

ALVAREZ V., V. H. Equilíbrio de formas disponíveis de fósforo e enxofre em dois Latossolos de Minas Gerais. 1974. 125f. Dissertação (Mestrado em Solos e Nutrição de Plantas) - Universidade Federal de Viçosa, Viçosa, MG, 1974.

BALIEIRO, F. C.; OLIVEIRA, I. G.; DIAS, L. E. Formação de mudas de Acacia holosericea e Acacia auriculiformis: Resposta à calagem, fósforo, potássio e enxofre. Revista Árvore, v.25, n.2, p.183-191, 2001.

BERNARDINO, D. C. S. et al. Influência da saturação por bases e da relação $\mathrm{Ca}: \mathrm{Mg}$ do substrato sobre o crescimento inicial de jacarandá-da-bahia (Dalbergia nigra (Vell.) Fr. All. Ex Benth.). Revista Árvore, v.31, n.4, p.567-573, 2007.

Revista Árvore, Viçosa-MG, v.35, n.5, p.983-995, 2011 
BRISSETTE, J. C. Summary of discussions about seedling quality. In: SOUTHERN NURSERY CONFERENCES, 1984, Alexandria. Proceedings... New Orleans: USDA. Forest Service, Southern Forest Experiment Station, 1984. p.127-128.

CARNEIRO, J. G. A. Variações na metodologia de produção de mudas florestais afetam os parâmetros morfofisiológicos que indicam a sua qualidade. Série Técnica FUPEF, n.12, p.1-40, 1983.

CARneiro, J. G. A. Produção e controle de qualidade de mudas florestais. Curitiba: UFPR/FUPEF, 1995. 451p.

CARVALHO, M. M. Ações visando recuperação de áreas de pastagens degradadas. In: ENCONTRO PARA CONSERVAÇÃO DA NATUREZA, 1., 1997, Viçosa. Anais... Viçosa, MG: CMCN/DEF/UFV, 1997. p.202-206.

CARVALHO, P. E. R. Espécies arbóreas brasileiras. Colombo: Embrapa Florestas, 2003. v.1. 1039p.

CECONI, D. E. et al. Crescimento de mudas de açoita-calavo (Luehea divaricata Mart.) sob influência da adubação fosfatada. Cerne, v.12, n.3, p.292-299, 2006.

CECONI, D. E. et al. Exigência nutricional de mudas de erva-mate (Ilex paraguariensis A. St.Hil.) à adubação fosfatada. Ciência Florestal, v.17, n.1, p.25-32, 2007.

CHAVES, L. L. B.; CARNEIRO, J. G. A.; BARROSO, D. G. Crescimento de mudas de Andenathera macrocarpa (Benth) Brenan (angico-vermelho) em substrato fertilizado e inoculado com rizóbio. Revista Árvore, v.30, n.6, p.911-919, 2006.

CRUZ, C. A. F. et al. Efeito de diferentes níveis de saturação por bases no desenvolvimento e qualidade de mudas de ipê-roxo (Tabebuia impetiginosa (Mart.) Standley). Scientia Forestalis, n.66, p.100-107, 2004.

CRUZ, C. A. F.; PAIVA, H. N.; GUERRERO, C. R. A. Efeito da adubação nitrogenada na produção de mudas de sete-cascas (Samanea inopinata (Harms) Ducke). Revista Árvore, v.30, n.4, p.537-546, 2006.

Revista Árvore, Viçosa-MG, v.35, n.5, p.983-995, 2011
DICKSON, A.; LEAF, A. L.; HOSNER, J. F. Quality appraisal of white spruce and white pine seedling stock in nurseries. Forestry Chronicle, v.36, n.1, p.10-13, 1960.

EUCLYDES, R. F. Manual de utilização do programa SAEG (Sistema para análises estatísticas e genéticas), Viçosa, MG:

Universidade Federal de Viçosa, 1997. 59p.

FERNANDES, L. A. et al. Crescimento inicial, níveis críticos de fósforo e frações fosfatadas em espécies florestais. Pesquisa Agropecuária Brasileira, v.35, n.6, p.1191-1198, 2000.

FERNANDES, A. R. et al. Efeito do fósforo e do zinco sobre o crescimento de mudas de freijó (Cordia goeldiana Huber.). Revista de Ciências Agrárias, n.37, p.123-131, 2002.

FERNANDES, A. R. et al. Crescimento e absorção de nutrientes por mudas de freijó (Crodia goeldiana Huber) em função de doses de fósforo e zinco. Revista Árvore, v.31, n.4, p.599-608, 2007.

GOMES, J. M. Parâmetros morfológicos na avaliação da qualidade de mudas de Eucalyptus grandis, produzidas em diferentes tamanhos de tubete e de dosagens de N-P-K. 2001. 126f. Tese (Doutorado em Ciência Florestal) - Universidade Federal de Viçosa, Viçosa, MG, 2001.

GOMES, K. C. et al. Crescimento de mudas de garapa em reposta à calagem e ao fósforo. Revista Árvore, v. 32, n.3, p. 387-394, 2008.

GONÇALVES, J. L. M. et al. Produção de mudas de espécies nativas: substrato, nutrição, sombreamento e fertilização. In: GONÇALVES, J. L. M.;

BENEDETTI, V. (Ed.). Nutrição e fertilização Florestal. Piracicaba: IPEF, 2000. p.309-350

GONÇALVES, E. O. et al. Crescimento de mudas de angico-vermelho (Anadenanthera macrocarpa (Benth.) Brenan) sob diferentes doses de macronutrientes. Revista Árvore, v.32, n.6, p.1029-1040, 2008.

LORENZI, H. Árvores brasileiras: manual de identificação e cultivo de plantas arbóreas nativas do Brasil. Nova Odessa: Plantarum, 1992. 352p. 
MARCHIORI, J. N. C. Dendrologia das angiospermas: leguminosas. Santa Maria: Universidade Federal de Santa Maria, 1997. 2000p.

MARQUES, V. B. et al. Efeito de fontes e doses de nitrogênio no crescimento de mudas de sabiá (Mimosa caesalpiniaefolia Benth.). Scientia Forestalis, n.71, p.77-85, 2006a.

MARQUES, V. B. et al.Efeito de fontes e doses de nitrogênio sobre o crescimento de inicial e qualidade de mudas de jacarandá-da-bahia (Dalbergia nigra (Vell.) Fr. All. Ex Benth.). Revista Árvore, v.30, n.5, p.725-735, 2006 b.

MARQUES, L. S. et al. Crescimento de mudas de jacaré (Piptadenia gonoacantha J. F. Macbr.) em diferentes tipos de solos e fontes e doses de nitrogênio. Revista Árvore, v.33, n.1, p.81-91, 2009.

MENDONÇA, A. V. R. et al. Exigências nutricionais de Myracroduon urundeuva Fr. All (Aroeira do Sertão). Revista Cerne, v.5, n.2, p.65-75, 1999.

NICOLOSO, F. T. et al. Nutrição mineral de mudas de grápia (Apuleia leiocarpa) em Argissolo Vermelho Distrófico Arênico: (1) Efeito da adubação NPK no crescimento. Ciência Rural, v.31, n.6, p.991-998, 2001.

PAIVA, H. N.; GOMES, J. M. Viveiros

florestais. 2.ed. Viçosa, MG: Universidade Federal de Viçosa, 2000. 69p. (Cadernos Didáticos, 72).
PEZZUTTI, R. V.; SCHUMACHER, M. V.; HOPPE, J. M. Crescimento de mudas de Eucalyptus globulus em resposta à fertilização NPK. Ciência Florestal, v.9, n.2, p.117-125, 1999.

REIS, M. G. F. et al. Exigências nutricionais de mudas de Dalbergia nigra (Vell.) Fr. Allem (Jacarandá-daBahia) produzidas em dois níveis de sombreamento. Revista Árvore, v.21, n.4, p.463-471, 1997.

RENÓ, N. B. et al. Limitações nutricionais ao crescimento inicial de quatro espécies arbóreas nativas em Latossolo Vermelho-Amarelo. Pesquisa

Agropecuária Brasileira, v.32, n.1, p.17-25, 1997.

SOUZA, C. A. M. et al. Crescimento em campo de espécies florestais em diferentes condições de adubações. Ciência Florestal, v.16, n.3, p.243-249, 2006.

SOUZA, P. H. et al. Influência da saturação por bases do substrato no crescimento e qualidade de mudas de Machaerium nictitans (Vell.) Benth. Revista Árvore, v.32, n.2, p.193-201, 2008.

TURRENT, F. A. Uso de una matriz mixta para la optimización de cinco a ocho factores controlables de la producción. Chapingo-México: Rama de Suelos, Colégio de Postgraduados, 1979. 65p. (Boletim Técnico, 6).

VENTURIN, N. et al. Adubação mineral do Angico-Amarelo (Peltophorum dubium (SPRENG.) TAUB.). Pesquisa Agropecuária Brasileira, v.34, n.3, p.441-448, 1999. 
